\title{
Multi-wavelength enhancement of silicon Raman scattering by nanoscale laser surface ablation
}

\author{
A. Merlen*1, A. Sangar ${ }^{1}$, P. Torchio², K. L. N. Deepak ${ }^{3}$, D. Grojo ${ }^{3}$, O. Utéza ${ }^{3}$, P. Delaporte ${ }^{3}$ \\ ${ }^{1}$ Université du Sud Toulon-Var, CNRS, IM2NP UMR 7334, Bâtiment R, BP 132, F-83957 \\ La Garde Cedex, France \\ ${ }^{2}$ Aix-Marseille Université, CNRS, IM2NP UMR 7334, Campus de Saint-Jérôme - Case 142, \\ F-13397 Marseille Cedex 20, France \\ ${ }^{3}$ Aix Marseille Université, CNRS, LP3 UMR 7341, 13288, Marseille, France \\ *merlen@univ-tln.fr
}

Submitted to Applied Surface Science

Keywords: Raman spectroscopy, Silicon, laser ablation

\begin{abstract}
In this paper, we produce nanoholes on a silicon surface by laser ablation. Those nanoholes lead to a yield enhancement of light-matter interaction. Performing Raman spectroscopy on silicon, an enhancement of its main Raman mode is observed: it is twice higher with the nanoholes compared to a flat surface. Such a feature appears whatever the excitation wavelength $(488,514.5$ and $632.8 \mathrm{~nm})$ and the laser power, revealing a broad band light-matter interaction enhancement. In addition, no change in the position and shape of the main Raman mode of silicon is observed, suggesting that no structural damages are induced by laser ablation. These results clearly demonstrate the potentiality of such nanostructures for the further development of silicon photonics.
\end{abstract}

\section{Introduction}

Trapping light using nanostructures is a powerful approach to improve light-matter interaction. This could lead to many applications, particularly in the field of photovoltaics [13]. Nowadays the huge development of optical nano-antennas [4] is an intensive field of research. However the exact interaction between those nanostructures and light is not totally clearly understood due to the multiple mechanisms interfering. In many cases light scattering can be enhanced. This feature has led to the development of surface enhanced spectroscopy, such as surface enhanced Raman spectroscopy (SERS [5]), surface enhanced fluorescence (SEF [6]) or surface enhanced infra-red absorption (SEIRA [7]). For all these techniques, the enhanced optical property of a nanostructured sample is used to detect a signal in conditions where this would normally not be possible due to a weak scattering cross section and a low amount of interacting molecules. For the above-mentioned techniques, the final signal intensity is an experimental sign of the enhanced scattering properties of the nanostructured sample. An enhancement factor (EF) can thus be calculated, even if it is absolutely necessary to be very cautious about its quantitative estimation and its real scientific meaning [5]. These techniques are extremely powerful and a single molecule detection has been reported [8-10]. They are usually all based on the giant electromagnetic resonance in the near field of metallic nanostructures due to plasmonic resonance. This is the case for instance with silver, gold or 
copper nanostructures. Depending on the plasmonic resonance, the intensity of the enhanced signal can thus strongly depends on the wavelength of the excitation and the scattered light. Even if this mechanism leads to a very high enhancement, its plasmonic origin induces a severe limitation: enhancement occurs only if the incoming light wavelength is included in a plasmon absorption band of the nanostructured sample. For broadband optical applications, it is therefore necessary to build plasmonic nanostructures with a broad absorption band, which can require sophisticated protocols (fractal nanostructures, use of a mix of different metals etc...).

Such mechanism is not the only one for the achievement of surface enhanced light scattering and several studies have already reported comparable enhancements without plasmonics. The use of silica microspheres enables also to obtain similar enhancements [11]. The precise mechanism is not fully explained but it appears that an optical resonance based on whispering-gallery mode plays a key role [12]. A lens effect can also occur [13]. In the case of nanostructured silicon (nanoparticles or microcavity) enhancements are also reported [14-16]. A good experimental proof of this effect is the evolution of the main silicon Raman mode. Due to its mechanism, Raman scattering can be considered as an experimental signature of light-matter interaction and is extensively used to study the optical near field enhancement. As a consequence, the light trapping in nanostructured samples induces an increase of Raman modes compared to a flat classical substrate. For silicon, the final intensity of its main Raman mode can therefore be considered as an experimental and quantitative proof of light trapping efficiency. Such specificity is of considerable interest for the development of silicon nanophotonics, in particular for photovoltaics.

Among all nanostructure architectures giving rise to an enhancement of light-matter interaction, the nanoholes appear as extremely promising [17]. In this paper we report Raman signal enhancement from silicon nanoholes. The silicon nanoholes are realized through laser ablation using silica nanospheres preliminary laid on the silicon substrate by means of the Langmuir-Blodgett technique. Those spheres act as lenses to focus the laser light. A network of nano-holes (typical diameter: $450 \mathrm{~nm}$ ) is thus created on the silicon surface. Such a sample is studied by Raman spectroscopy using excitation wavelengths at $488 \mathrm{~nm}, 514.5 \mathrm{~nm}$ and $632.8 \mathrm{~nm}$. For each wavelength, measurements are performed with various laser powers. A clear enhancement of the silicon Raman mode is observed, whatever the excitation wavelength and the laser power are. Our results confirm the possibility offered by such nanostructured sample to highly increase light-silicon interaction.

\section{Experimental}

Oriented (100) silicon wafers (from Siltronix) are used in the experiments. The wafers are polished on a single side and cut into $\approx 2 \times 2 \mathrm{~cm}^{2}$ area samples. They are sonicated in water and ethanol for 30 minutes, and further treated with a plasma torch (Acxys technologies) in order to remove any residual contamination and to increase the wettability of the surface.

Afterwards, silica spheres with radii of $500 \mathrm{~nm}$ and low size dispersion (polydispersity index PDI < 0.2) are mixed with ethanol $(40 \mathrm{mg} / \mathrm{mL})$. A Langmuir-Blodgett (LB) film deposition machine (KSV-Nima, model Mini) equipped with a surface tension balance is employed to grow a close-packed sphere monolayer on the Si substrates. We choose to work with $\mathrm{C} 18$ functionalized commercial spheres (from Micromod) as they are strongly hydrophobic, enabling the self-assembly of spheres at the Air/Water Interface without the use of chemicals $[18,19]$ that would modify the spheres or the LB subphase. The microsphere solution is carefully spread by small droplets $(\mu \mathrm{L})$ at the surface of water which is then compressed until the surface pressure reaches $15 \mathrm{mN} / \mathrm{m}$. The monolayer is then transferred to the silicon surface at a dipping speed of $5 \mathrm{~mm} / \mathrm{minute}$ while the surface pressure is maintained at a constant level by progressive automated compression. 
The microsphere 2D-arrays obtained on silicon are irradiated by laser pulses provided by an $\operatorname{ArF}\left(\lambda_{\text {laser }}=193 \mathrm{~nm}\right.$ ) laser source (from Lambda Physik, LPX220i). The laser pulse duration is $15 \mathrm{~ns}$, and the experiments are performed at normal incidence. The spheres act as near-field focusing elements producing a periodic assembly of sub-diffraction limit light spots (also called photonic nanojets) hitting the silicon surface [20]. When modest laser fluences are used, the substrate is ablated only locally at the tip of the photonic nanojets leaving behind an array of nanocraters in a simple, dry and fast single laser processing step [21].

Obviously the quality of the periodic structuring depends on the quality of the arrangement resulting from the sphere assembly, the level of monodispersity of the spheres and also on the laser dose impinging the substrate [22]. The laser-pulse energy was varied with the aid of a manually operated beam attenuator (from Optec, AT4030).

In previous works [23], parallel and long range ( > $\left.\mathrm{mm}^{2}\right)$ structuring at the mesoscopic scale $(100 \mathrm{~nm}-1 \mu \mathrm{m})$ has been demonstrated on silicon samples showing the interest of this approach. We also studied the degree of control in surface structuring while varying the laser fluence and number of shots. The results relied on statistical analyses of the created features (in the range 100-1000 $\mathrm{nm}$ ) observed by optical and scanning electron microscopy (SEM, JEOL JSM-6390) [23]. In the present case, the templates are prepared with 10 laser shots at a laser fluence maintained at $0.55 \mathrm{~J} / \mathrm{cm}^{2}$. This sequence is found to provide high quality structures for experiments performed at ambient air. The structures rely on the local ablations induced by the first pulse which ejects the microsphere and leaves a small hole behind it.

Cleaning as well as local ablation of the resulting products around the holes is realized with the following laser pulses. Similar results could be likely obtained with a single laser pulse if the interaction were performed in a low pressure environment to avoid redeposition.

The morphology of the silicon templates used for the optical experiments is analyzed using tapping-mode atomic force microscopy (AFM, PSIA XE-100). An AFM image of the laserimpacted silicon sample can be seen in figure 1. A hexagonal network of holes is formed after laser ablation. The pitch is $1 \mu \mathrm{m}$ imposed by the sphere radius. The holes have a typical diameter of $440 \mathrm{~nm}$ and a deepness of $230 \mathrm{~nm}$.

Raman measurements are performed using a Horiba Labram HR800 spectrometer. Three different laser excitations wavelengths are used: $632.8 \mathrm{~nm}$ (from a HeNe laser), $514.5 \mathrm{~nm}$ and $488 \mathrm{~nm}$ (from an Ar laser). The laser beam is focused on the sample through a x100 objective (Numerical Aperture of 0.9). The laser spot size is similar to the typical diameter of the nanoholes. As a consequence, the signal relies on an individual nanohole for each Raman measurement. The use of different laser excitation wavelengths insures a rather broadband study of the optical response of the sample. The laser power varies between $1 \mu \mathrm{W}$ and 3.6 $\mathrm{mW}$. 


\section{Results and discussion}

a)

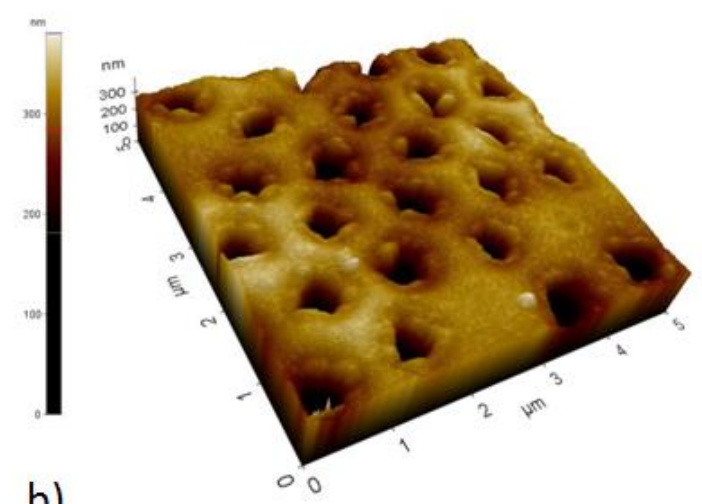

b)

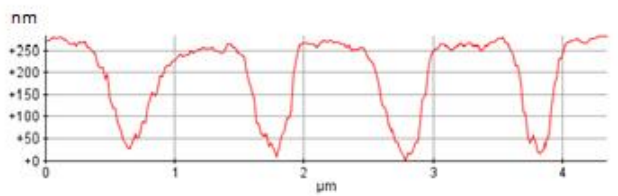

Fig. 1. AFM image of a laser-impacted silicon sample. a) General 3D view and b) typical line profile of the nanoholes array.

For the different laser excitation wavelengths, two kinds of Raman measurements were performed: one with the laser focused on a hole and the other one on a flat part of the sample between the holes. Despite the high power laser irradiation, the Raman signal coming from the flat part of the laser-impacted silicon sample is identical to the one from a standard flat silicon sample (position of the peak, full half-width maximum and intensity). The recorded Raman spectra of the laser-impacted silicon samples can be seen in figure 2 . The single mode observed at $520.7 \mathrm{~cm}^{-1}$ arises from the first-order Raman scattering of the longitudinal optical (LO) and the transverse optical (TO) phonon modes which are degenerated at the $\Gamma$-point (phonon wavevector $\mathrm{q} \approx 0$ near the center of the Brillouin zone). 

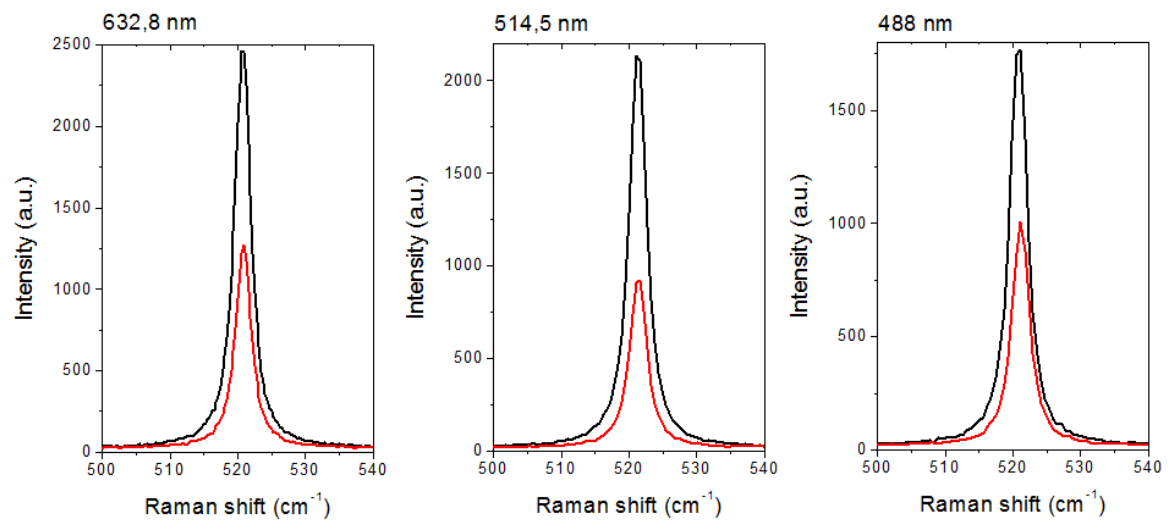

Fig. 2. Raman spectra of laser-impacted silicon samples for the three laser excitations wavelengths: $488 \mathrm{~nm}, 514.5 \mathrm{~nm}$ and $632.8 \mathrm{~nm}$. Two spectra are reported for each wavelength: one with the laser focused on a hole (black curve) and the other one on a flat part of the same sample (red curve).

Surprisingly the position of the peak and its profile are not changed after laser structuring of the sample surface. Such a feature suggests that the laser irradiation of the silicon surface induces little strain on the silicon lattice. Indeed it was reported that any significative strain should induce a shift and a broadening of the mode [24]. This is not observed here whatever the laser excitation wavelength is. It cannot be excluded that such stress could appear at the top surface, but that was not observed here due to the light penetration in silicon. If such stress occurs, it would happen at the top surface of silicon and would not be detected by classical Raman spectroscopy. Similar Raman measurements were performed on silicon samples ablated with much higher laser fluences for structuring compared to that used in the current study. The Raman mode of these high power impacted-laser silicon samples was shifted and strongly broadened, confirming the high sensitivity of our own measurement process to these issues. Such experimental feature has already been reported in previous studies [25, 26]. Our structuring method is remarkable to prove that, despite the surface structuring is carried out in the ablation laser regime, no significant change in the silicon structure in nanoholes is detected by Raman spectroscopy.

Even if the position of the Raman peak and its profile are not affected by laser structuring, it appears clearly that its intensity strongly depends on the structuring of the silicon surface: for any wavelengths the intensity is approximately twice as much for the nanostructured area compared to a flat zone. Depending on the experimental conditions (laser wavelength, laser power, light focalization, irradiated area) the intensity enhancement varies from 1.3 to 2.5 with a typical value around 2 . 


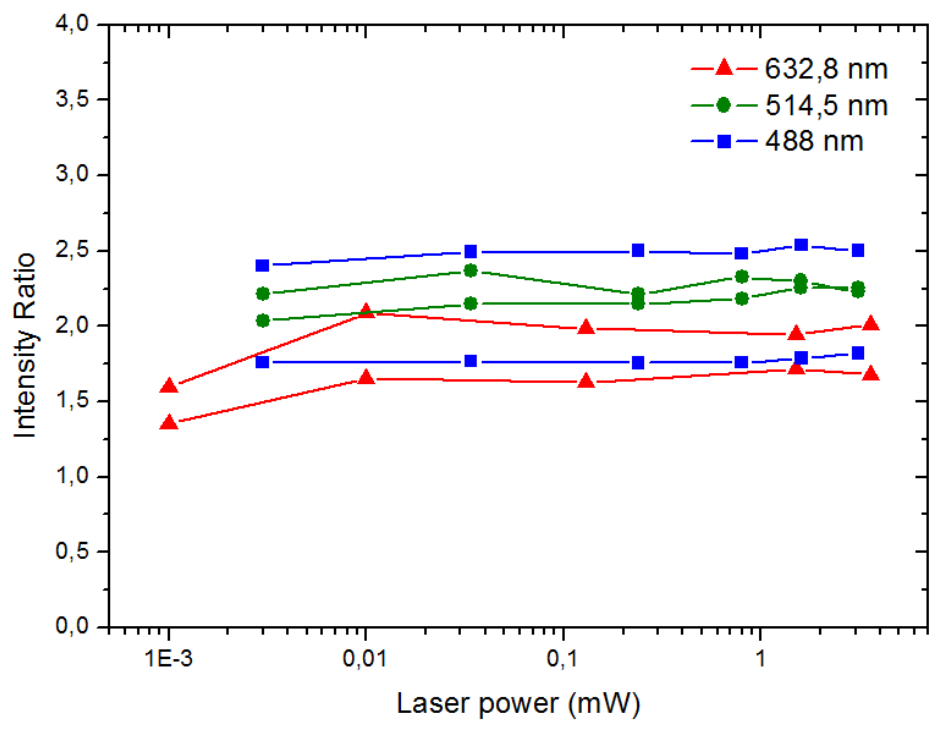

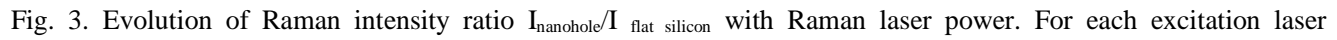
wavelength two series of measurements are reported.

In figure 3 the intensity ratio $I_{\text {nanohole }} / I_{\text {flat silicon }}$ for the three excitation laser wavelengths and different Raman laser powers can be seen. Except for low power laser illumination (for which the Raman signal is weak and the evaluation of Raman intensity is rather imprecise), this enhancement factor is almost independent of the laser power. In addition it does not vary significantly with the excitation wavelength value. The independence of the enhancement with laser excitation wavelength and laser power is remarkable. To our knowledge this is the first time that such behavior is reported. This result is an extremely important aspect to promote the use of nanoholes in the improvement of broadband silicon photonics.

The origin of this enhancement needs to be explained. As silicon nanostructures do not involve any plasmonic resonance for such wavelengths, this enhancement obeys a nonplasmonic mechanism. This is confirmed by the independence of the enhancement versus wavelength: for plasmonic nanostructures with spectral narrow-band resonance, enhancement is strongly dependant with the excitation wavelength [27].

In Raman spectroscopy (and generally for any light-matter interaction process), it is necessary to take into account the amount of probed matter when comparing two different samples. The surface ratio between a flat round and a curved round probed surface is 2 , which is noticeable close to the enhancement factors reported in figures 2 and 3. This ratio can also vary depending on the exact shape of the hole. According to AFM images, the shape of our holes is not so far from a spherical hole. In addition to the surface increase, the whole volume where light-matter interaction occurs has to be considered to discuss the origin of the enhancement. Optical absorption length in silicon strongly depends on the wavelength used for Raman measurements: it is typically $495 \mathrm{~nm}$ at $488 \mathrm{~nm}, 680 \mathrm{~nm}$ at $514 \mathrm{~nm}$ and $2700 \mathrm{~nm}$ at $632 \mathrm{~nm}$ [28]. But we demonstrated that the intensity ratio between the flat and the nanostructured sample is independent of the wavelength (see figures 2 and 3). This suggests that the increase of surface or volume interaction is not the only mechanism responsible for the enhancement of Raman scattering.

Liu et al. [15] studied electrochemically roughened silicon substrates. They attributed the Raman scattering enhancement to two contributions: one due to an electromagnetic cavity resonance and the other to a resonance arising from silicon nanoparticles. In our Raman 
experiments, no significant shift and broadening of the silicon mode are observed suggesting that no silicon nanoparticles are formed during the ablation laser process. Finally the enhancement observed herein very probably arises from the cavity resonance only. It must be noticed that such electromagnetic cavity resonance which induces a high Raman enhancement was also reported for silicon nanowires [29] and nanocones [30]. And this resonance mechanism occurring with silicon nanoholes should have broad-band properties as no significant difference in the final enhancement is observed among our three excitation wavelengths.

Another feature should also be taken into account: a light trapping effect. Multiple light reflections and light scattering could happen in the nanoholes, giving rise to multiple Raman scattering and an increase of the final intensity of the Raman mode. Such multiple reflections were already reported with inverted pyramidal nanostructures [1] which are considered as extremely promising anti-reflective treatment for silicon. Korsunska et al. [31] explained also the Raman enhancement observed with macro- and nanoporous silicon by multiple absorption of the probe light beam scattered and reflected inside pores. This light trapping effect plays certainly a role in the final enhancement observed herein.

\section{Conclusion}

We demonstrated through Raman spectroscopy that the use of nanoholes can enhance lightsilicon interactions. Moreover this enhancement occurs without any clear sign of structural damages induced by laser irradiation during nanoholes fabrication. Microsphere mediated laser ablation can be an efficient method to prepare dense arrays of appropriate nanoholes. The enhancement of the Raman signal intensity coming from the structured area of a silicon substrate compared to the flat part is reported between 488 and $632.8 \mathrm{~nm}$. This amplified intensity ratio is independent of the excitation wavelength and power of the Raman laser probe. Such an enhancement is induced by the combination of several mechanisms: a broadband optical resonance, an increase of the interaction surface and multiple scattering in cavities. The relative contribution of each phenomenon remains unclear and will require further study. The global enhancement of optical properties of nanostructured silicon with nanoholes is extremely promising for the future development of silicon Nanophotonics. This material engineering could also be applied to other optically active materials. It is confirmed that Raman spectroscopy is an efficient tool to study light-matter interaction in nanostructures.

\section{Acknowledgements}

The authors acknowledge the Agence Nationale de la Recherche (ANR) for financial support under the CARIOCA (number 2010-JCJC-918-01) and FELINS-ANR-10-BLAN-946 projects.

[1] H.H. Cheng, Y.-Y. Chang, J.-Y. Chu, D.-Z. Lin, Y.-P. Chen, J.-H. Li, Light trapping enhancements of inverted pyramidal structures with the tips for silicon solar cells, Appl. Phys. Lett., 101, 141113 (2012).

[2] N.F. Fahim, B.H. Jia, Z.R. Shi, M. Gu, Simultaneous broadband light trapping and fill factor enhancement in crystalline silicon solar cells induced by $\mathrm{Ag}$ nanoparticles and nanoshells, Optics Express, 20, A694-A705 (2012).

[3] S. Vedraine, P. Torchio, A. Merlen, J. Bagierek, F. Flory, A. Sangar, L. Escoubas, Optical characterization of organic blend films integrating metallic nanoparticles, Sol. Energy Mater. Sol. Cells, 102, 31-35 (2012).

[4] L. Novotny, Optical antennas tuned to pitch, Nature, 455, 887-887 (2008).

[5] P.G. Etchegoin, E.C. Le Ru, Principles of surface-enhanced Raman spectroscopy and related plasmonic effects, Elsevier (2009). 
[6] E. Fort, S. Gresillon, Surface enhanced fluorescence, J. Phys. D-Appl. Phys., 41, 013001 (2008).

[7] R.F. Aroca, D.J. Ross, C. Domingo, Surface-enhanced infrared spectroscopy, Applied Spectroscopy, 58, 324A-338A (2004).

[8] S.M. Nie, S.R. Emery, Probing single molecules and single nanoparticles by surfaceenhanced Raman scattering, Science, 275, 1102-1106 (1997).

[9] P.G. Etchegoin, E.C. Le Ru, A perspective on single molecule SERS: current status and future challenges, Physical Chemistry Chemical Physics, 10, 6079-6089 (2008).

[10] K. Kneipp, Y. Wang, H. Kneipp, L.T. Perelman, I. Itzkan, R. Dasari, M.S. Feld, Single molecule detection using surface-enhanced Raman scattering (SERS), Physical Review Letters, 78, 1667-1670 (1997).

[11] M.S. Anderson, Nonplasmonic surface enhanced Raman spectroscopy using silica microspheres, Appl. Phys. Lett., 97, 131116 (2010).

[12] L.K. Ausman, G.C. Schatz, Whispering-gallery mode resonators: Surface enhanced Raman scattering without plasmons, J. Chem. Phys., 129, 054704 (2008).

[13] A. Desmedt, D. Talaga, J.L. Bruneel, Enhancement of the Raman scattering signal due to a nanolens effect, Applied Spectroscopy, 61, 621-623 (2007).

[14] L.A. Kuzik, V.A. Yakovlev, G. Mattei, Raman scattering enhancement in porous silicon microcavity, Appl. Phys. Lett., 75, 1830-1832 (1999).

[15] F.M. Liu, B. Ren, J.H. Wu, J.W. Yan, X.F. Xue, B.W. Mao, Z.Q. Tian, Enhanced-Raman scattering from silicon nanoparticle substrates, Chemical Physics Letters, 382, 502-507 (2003).

[16] L. Sirleto, M. Antonietta Ferrara, T. Nikitin, S. Novikov, L. Khriachtchev, Giant Raman gain in silicon nanocrystals, Nature communications, 3, 1220-1220 (2012).

[17] V. Pellegrini, A. Tredicucci, C. Mazzoleni, L. Pavesi, Enhanced optical-properties in porous silicon microcavities, Physical Review B, 52, 14328-14331 (1995).

[18] L.M. Goldenberg, J. Wagner, J. Stumpe, B.R. Paulke, E. Gornitz, Simple method for the preparation of colloidal particle monolayers at the water/alkane interface, Langmuir, 18, 5627-5629 (2002).

[19] M. Bardosova, M.E. Pemble, I.M. Povey, R.H. Tredgold, The Langmuir-Blodgett approach to making colloidal photonic crystals from silica spheres, Adv. Mater., 22, 31043124 (2010).

[20] D. Grojo, L. Charmasson, A. Pereira, M. Sentis, P. Delaporte, Monitoring photonic nanojets from microsphere arrays by femtosecond laser ablation of thin films, J. Nanosci. Nanotechnol., 11, 9129-9135 (2011).

[21] K. Piglmayer, R. Denk, D. Bauerle, Laser-induced surface patterning by means of microspheres, Appl. Phys. Lett., 80, 4693-4695 (2002).

[22] D. Grojo, L. Boarino, N. De Leo, R. Rocci, G. Panzarasa, P. Delaporte, M. Laus, K. Sparnacci, Size scaling of mesoporous silica membranes produced by nanosphere mediated laser ablation, Nanotechnology, 23, 485305 (2012).

[23] D.Grojo, K.L.N. Deepak, L. Charmasson, P. Delaporte, O. Utéza, A. Merlen, A. Sangar, P. Torchio, Long range nanostructuring of silicon surfaces by photonic nanojets from microsphere Langmuir films, J. Phys. D: Applied Physics, 46, 145102 (2013)

[24] P. Kumar, M.S.R.N. Kiran, Nanomechanical properties of silicon surfaces nanostructured by excimer laser, Science and Technology of Advanced Materials, 11, 025003 (2010).

[25] J. Reif, O. Varlamova, M. Bounhalli, M. Muth, T. Arguirov, Nanostructure formation upon femtosecond ablation from silicon: Effect of double pulses, Appl. Surf. Sci., 258, 94919495 (2012).

[26] J. Bonse, K.W. Brzezinka, A.J. Meixner, Modifying single-crystalline silicon by femtosecond laser pulses: an analysis by micro Raman spectroscopy, scanning laser microscopy and atomic force microscopy, Appl. Surf. Sci., 221, 215-230 (2004). 
[27] A. Merlen, V. Gadenne, J. Romann, V. Chevallier, L. Patrone, J.C. Valmalette, Surface enhanced Raman spectroscopy of organic molecules deposited on gold sputtered substrates, Nanotechnology, 20, 215705 (2009).

[28] E.D. Palik, Handbook of optical constants of solids, (1991).

[29] M. Khorasaninejad, N. Dhindsa, J. Walia, S. Patchett, S.S. Saini, Highly enhanced Raman scattering from coupled vertical silicon nanowire arrays, Appl. Phys. Lett., 101, 173114 (2012).

[30] L.Y. Cao, B. Nabet, J.E. Spanier, Enhanced raman scattering from individual semiconductor nanocones and nanowires, Phys. Rev. Lett., 96, 157402 (2006).

[31] N. Korsunska, B. Bulakh, B. Jumayev, L. Khomenkova, V. Yukhymchuk, T. Torchynska, Raman scattering characterization of macro- and nanoporous silicon, Appl. Surf. Sci., 243, 30-35 (2005). 